\title{
Preparing low-copy number plasmid DNA using the ZymoPURE ॥ Plasmid Maxiprep Kit
}

\section{Improving yield for low-copy number plasmid DNA}

\author{
Zymo Research Corporation \\ *Correspondence: SBiondi@zymoresearch.com
}

\section{Introduction}

Plasmids differ significantly in copy number due to their origin of replication, size, and associated insert. Several plasmids contain mutations that allow them to reach very high-copy numbers within the bacterial cell. However, depending on the objectives of the experiment or the nature of the cloned insert, it is not always possible to use high-copy number plasmid DNA.

Preparing low-copy number plasmid DNA can become a significant burden for applications that require large quantities of highly concentrated, endotoxin-free plasmid DNA, such as transfections and in vivo genetic manipulations. In these cases, it requires either scaling up the culture, performing multiple preparations, and/or concentrating the plasmid following purification.

Zymo Research has developed an innovative protocol for the ZymoPURETM II Maxiprep Kit that enables purification of high quantities of
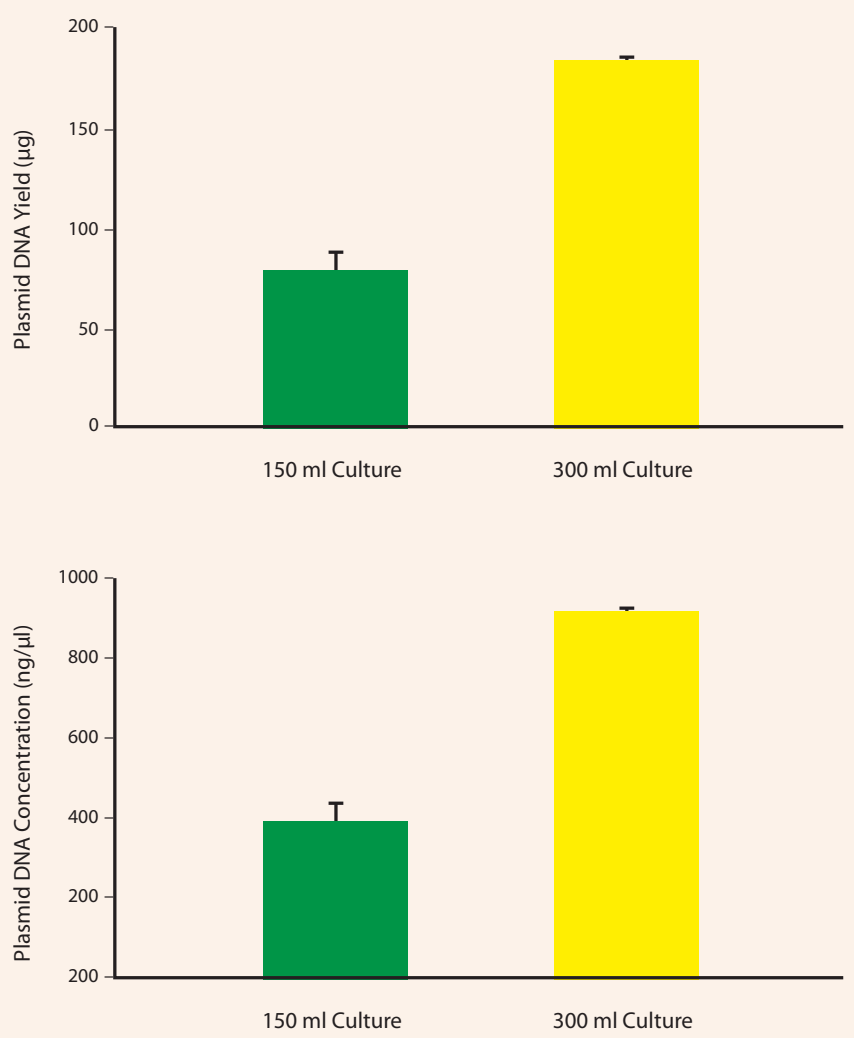

Figure 1. Processing a higher volume of culture dramatically increases yield. The low-copy number plasmid pBR322 was isolated from either $150 \mathrm{ml}$ or $300 \mathrm{ml}$ of JM109 E. coli culture grown overnight using the ZymoPURETM II Maxiprep Kit and a mod-ified protocol for low-copy number plasmid DNA. Plasmid DNA yield (upper panel) and concentration (lower panel) was determined by measuring the absorbance of the eluted plasmid DNA at $260 \mathrm{~nm}$ using a Nanodrop 2000 Spec-trophotometer (Thermo Fisher). Shown are means \pm SD of two preparation. plasmid DNA from low-copy number plasmids. Using this protocol, researchers can quickly and efficiently isolate highly concentrated, transfection-grade, low-copy number plasmid DNA at workable concentrations in $\sim 25$ minutes.

\section{Methods}

\section{Bacterial Culture}

A $2 \mathrm{ml}$ JM109 E. coli culture harboring the low-copy number plasmid pBR322 (4,361 bp) was grown for 8 hours and then diluted $1: 1,000$ into $150 \mathrm{ml}$ or $300 \mathrm{ml}$ of LB broth + ampicillin in a 1 liter or 2 liter Erlenmeyer flask. The expanded cultures were grown for 18 hours at $37^{\circ} \mathrm{C}$ with vigorous shaking at 250 rpm and the bacterial cells were collected by $\mathrm{C} \neg$-entrifugation at $5,000 \times \mathrm{g}$ for 10 minutes in a $250 \mathrm{ml}$ centrifugation bottle.

\section{Plasmid DNA Purification}

The bacterial cell pellet was resuspended in $14 \mathrm{ml}$ of ZymoPURETM $\mathrm{P} 1$ and then lysed by the addition of $14 \mathrm{ml}$ of ZymoPURETM P2, followed by gently inverting the solution 10 times and incubating the lysate for 5 minutes at room temperature.

Following lysis, the solution was neutralized by the addition of $14 \mathrm{ml}$ of ZymoPURE'M P3 and mixed by gentle inversion until the solution turned completely yellow and a greenish-yellow precipitate formed, indicating complete neutralization. The lysate debris was removed by centrifugation for 10 minutes at 5,000 xg and then further clarified by passing the supernatant through a ZymoPURETM syringe filter until approximately $35 \mathrm{ml}$ of cleared lysate was recovered.

To the $35 \mathrm{ml}$ of clarified lysate, $14 \mathrm{ml}$ of ZymoPURETM binding buffer was added, and the solution was mixed by inverting 10 

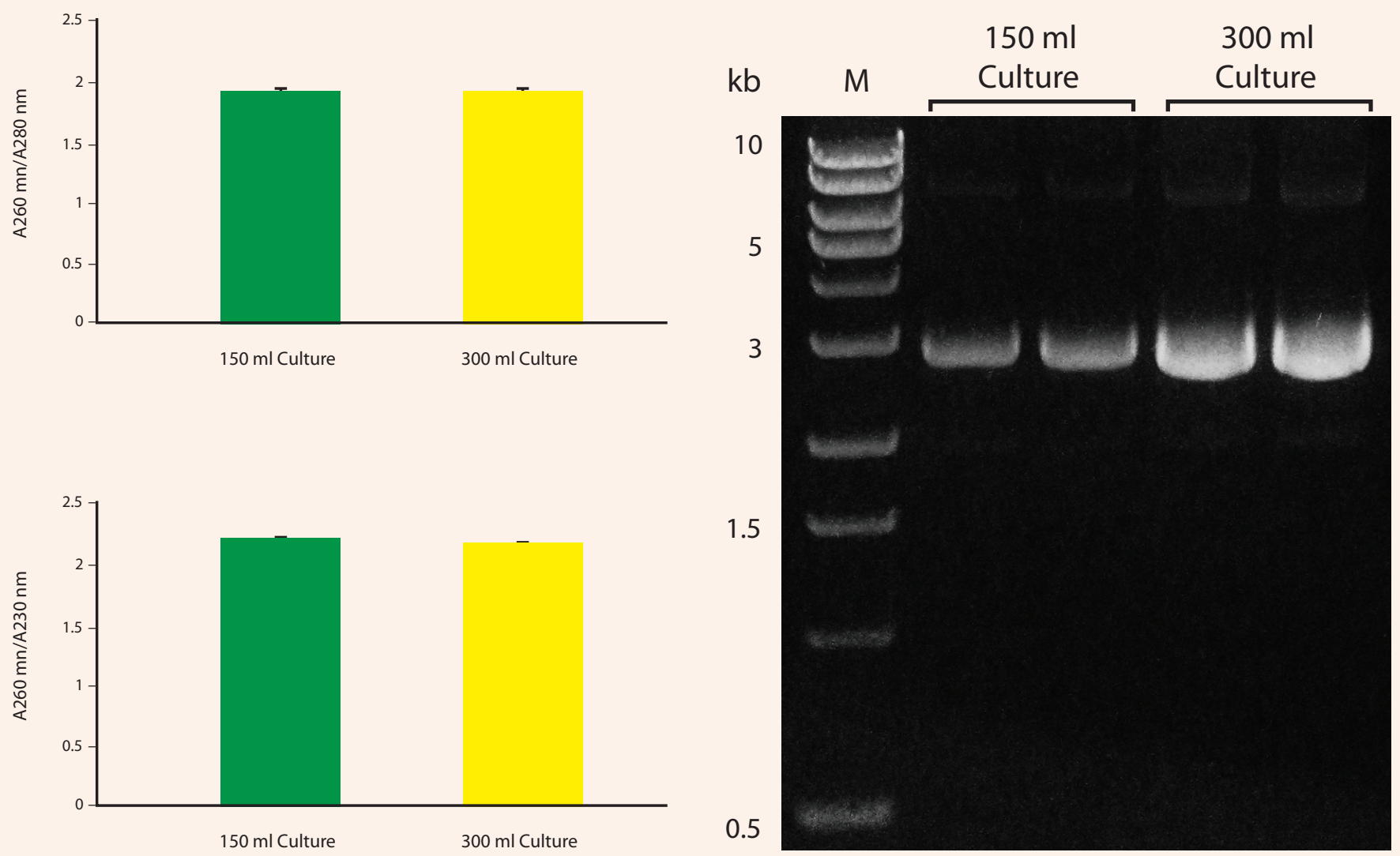

Figure 2. Processing a higher volume of culture does not adversely affect plasmid DNA quality. Plasmid DNA purity was determined by measuring the absorbance of the eluted plasmid DNA at wavelengths $230 \mathrm{~nm}, 260$ $\mathrm{nm}$, and $280 \mathrm{~nm}$ using a Nanodrop $2000 \mathrm{Spec} \neg$ trophotometer (Thermo Fisher) and then calculating the ratio of A260/A280 (upper panel) and A260/A230 (lower panel). Shown are mean \pm SD of two preparations.

Figure 3. Plasmid DNA is free of contaminating RNA and genomic DNA. Plasmid DNA quality was assessed by agarose gel electrophoresis of $1 \mu \mathrm{l}$ of eluted plasmid DNA. M: ZR 1 kb DNA Marker (Zymo Research).

times. A Zymo-SpinTM V-P column assembly was attached to an EZ-Vac'M vacuum manifold and the binding buffer and lysate mixture was poured into the column assembly. A vacuum pressure of $400 \mathrm{~mm} \mathrm{Hg}$ was applied until the solution passed completely through the column.

The $50 \mathrm{ml}$ reservoir was removed from the top of the Zymo-Spin ${ }^{\top M} \mathrm{~V}-\mathrm{P}$ column assembly. The column was then washed with $5 \mathrm{ml}$ of ZymoPURETM Wash 1. This was followed by two additional washes with $5 \mathrm{ml}$ of ZymoPURETM Wash 2 . The $15 \mathrm{ml}$ conical reservoir was discarded, and residual wash buffer was removed by placing the Zymo-Spin ${ }^{\mathrm{TM}} \mathrm{V}-\mathrm{P}$ column in a collection tube and performing centrifugation at $10,000 \times \mathrm{g}$ for 1 minute.

The Zymo-Spin ${ }^{\mathrm{TM}} \mathrm{V}$-P column was then placed into a clean $1.5 \mathrm{ml}$ microcentrifuge tube and $200 \mu \mathrm{l}$ of ZymoPURETM elution buffer was added directly to the column matrix. The column was incubated at room temperature for 2 minutes and then centrifuged at $10,000 \times \mathrm{g}$ for 1 minute in order to collect the eluate. The resulting eluate was placed into the EndoZero ${ }^{\mathrm{TM}}$ spin column, incubated at room temperature for 2 minutes, and then centrifuged at $5,000 \times \mathrm{g}$ for 1 minute.

\section{Results}

Preparing low-copy number plasmid DNA for molecular biology techniques that require highly concentrated, ultra-pure plasmid DNA can be cumbersome. To increase yield for low-copy plasmids, modifications to the standard ZymoPURETM II Maxiprep Kit protocol were made. These include doubling the volume of culture to be processed, inverting additional times after adding ZymoPURE ${ }^{\text {TM }}$ P2, increasing the standard lysis incubation by 3 minutes, and pelleting most of the lysate debris prior to clearing with the ZymoPURE ${ }^{\mathrm{TM}}$ syringe filter. With these modifications, approximately twice as much low-copy number pBR322 plasmid was isolated (Fig. 1). Furthermore, by reducing the elution volume from $400 \mu \mathrm{l}$ to $200 \mu \mathrm{l}$, the concentration of pBR322 in the eluate approaches $1 \mu \mathrm{g} / \mu \mathrm{l}$ when isolated from a $300 \mathrm{ml}$ culture volume (Fig. 1). This ensures the plasmid DNA concentration is suitable for downstream applications.

ZymoPURETM II Maxiprep Kit and a mod-ified protocol for low-copy number plasmid DNA. Plasmid DNA yield (upper panel) and concentration (lower panel) was determined by measuring the absorbance of the eluted plasmid DNA at 260 nm using a Nanodrop 2000 Spec-trophotometer (Thermo Fisher). Shown are means \pm SD of two preparation. 
In addition, the resulting isolated plasmid DNA achieved A260/A280 and A260/A230 ratios of $>1.8$, ensuring the plasmid preparations are free of contaminating salts and protein, regardless of input volume (Fig. 2). Further analysis by agarose gel electrophoresis demonstrated that the purified plasmid is predominately supercoiled and does not contain RNA or genomic DNA (Fig. 3).

\section{Conclusions}

By using a modified protocol, twice as much low-copy number plasmid DNA can be purified in approximately 25 minutes with the ZymoPURE'M II Plasmid Maxiprep Kit. Moreover, the isolated plasmid DNA is ultra-pure and highly concentrated making it ideal for transfection and other sensitive downstream applications.

Biotechniques 65: 365-367 (December 2018) doi: 10.2144/btn-2018-2013

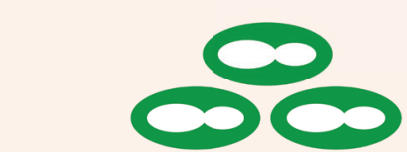

ZYMO RESEARCH

The Beauty of Science is to Make Things Simple ${ }^{\circ}$ 